USC-94-013

hep-th/9408004

\title{
The boundary sine-Gordon theory: classical and semi-classical analysis
}

\author{
H. Saleur*, S. Skorik and N.P. Warner \\ Physics Department \\ University of Southern California \\ University Park \\ Los Angeles, CA 90089-0484.
}

\begin{abstract}
We consider the sine-Gordon model on a half-line, with an additional potential term of the form $-M \cos \frac{\beta}{2}\left(\varphi-\varphi_{0}\right)$ at the boundary. We compute the classical time delay for general values of $M, \beta$ and $\varphi_{0}$ using $\tau$-function methods and show that in the classical limit, the method of images still works, despite the non-linearity of the problem. We also perform a semi-classical analysis, and find agreement with the exact quantum S-matrix conjectured by Ghoshal and Zamolodchikov.
\end{abstract}

* Packard Fellow

July, 1994 


\section{Introduction}

There are a wealth of applications of 1+1-dimensional quantum field theories defined upon the half-line. Such theories generically have some potential term at the boundary, and are thus often referred to as "boundary field theories." Amongst other things, they have been used to describe dissipative quantum mechanics [1], Kondo effects [2], and quantum wires [3,4]. The exemplar of such theories is the sine-Gordon model, whose Lagrangian is given by:

$$
L=\frac{1}{2} \int_{0}^{\infty}\left[\left(\partial_{t} \varphi\right)^{2}-\left(\partial_{x} \varphi\right)^{2}+g \cos \beta \varphi\right] d x+M \cos \frac{\beta}{2}\left(\varphi(x=0)-\varphi_{0}\right) .
$$

The massless limit for $\beta^{2}=8 \pi$ was considered in [5], and for generic values of $\beta^{2}$ in [6].

The quantum version of the sine-Gordon model was considered by Ghoshal and Zamolodchikov [7], who conjectured its integrability and an exact S-matrix. Our purpose here is to discuss these two aspects in the classical and semi-classical limits, generalizing the well known bulk analysis [8,3,9] to the boundary problem (1.1).

We establish the classical integrability in the next section by using the bulk sineGordon theory and the method of images. Although such a method might not be expected to work, a priori, due to the non-linearity, it turns out that the boundary condition inherited from (1.1) can be realized, for the boundary reflection of a single soliton, by using a three soliton solution of the bulk problem. We compute the classical time delay for the most general values of the parameters $M$ and $\phi_{0}$. The semi-classical analysis is performed in the third section of this paper. We find agreement with the $\beta \rightarrow 0$ limit of the results in [7].

\section{The classical solutions}

For a conformal field theory, or for any linear partial differential equation, the simplest method of dealing with a boundary is to use some version of the method of images [10]. One of the purposes of this section is to describe how this technique can also be applied to the classical sine-Gordon equation on the semi-infinite interval. Indeed, we will show that this method replicates the soliton scattering from a boundary with the most general boundary conditions consistent with integrability [7]. 


\subsection{The $\tau$-functions}

After appropriate rescaling of the field and the coupling constant we have, for the classical problem associated with the bulk part of (1.1), the sine-Gordon equation

$$
\phi_{t t}-\phi_{x x}=-\sin (\phi)
$$

where $\beta \varphi \equiv \phi$. On the interval $[0, \infty)$ it was argued in [7] that the most general boundary conditions consistent with integrability were:

$$
\left.\partial_{x} \phi\right|_{x=0}=\left.M \sin \left(\frac{1}{2}\left(\phi-\phi_{0}\right)\right)\right|_{x=0}
$$

for some constants $M$ and $\phi_{0}$, corresponding to the boundary term in (1.1).

On the infinite interval, $(-\infty, \infty)$, the classical multi-soliton solution to the sineGordon equation is well known [1]. It is usually expressed as:

$$
\phi(x, t)=4 \arg (\tau) \equiv 4 \operatorname{artan}\left(\frac{\mathcal{I} m(\tau)}{\mathcal{R} e(\tau)}\right)
$$

where the $\tau$-function for the $N$-soliton solution is:

$$
\begin{array}{r}
\tau=\sum_{\mu_{j}=0,1} e^{\frac{i \pi}{2}\left(\sum_{j=1}^{N} \epsilon_{j} \mu_{j}\right)} \exp \left[-\sum_{j=1}^{N} \frac{1}{2} \mu_{j}\left[\left(k_{j}+\frac{1}{k_{j}}\right) x+\left(k_{j}-\frac{1}{k_{j}}\right) t-a_{j}\right]\right. \\
\left.+2 \sum_{1 \leq i<j \leq N} \mu_{i} \mu_{j} \log \left(\frac{k_{i}-k_{j}}{k_{i}+k_{j}}\right)\right] .
\end{array}
$$

The parameters $k_{j}, a_{j}$ and $\epsilon_{j}$ have the following interpretations. The velocity of the $j^{\text {th }}$ soliton is given by:

$$
v_{j}=\left(\frac{k_{j}^{2}-1}{k_{j}^{2}+1}\right) .
$$

(Note that $v_{j}$ is positive for a left-moving soliton.) The $a_{j}$ represent the initial positions of each of the solitons, and $\epsilon_{j}=+1$ if the $j^{\text {th }}$ soliton is a kink, while $\epsilon_{j}=-1$ if it is an anti-kink. The rapidity, $\theta$, of the soliton is defined by $k=e^{\theta}$, and we have normalized the soliton masses to unity (in further discussion, the words "soliton" and "kink" will be used synonymously).

It is fairly obvious how to get a single soliton solution on $[0, \infty)$ with either $\left.\phi\right|_{x=0}=0$ or $\left.\partial_{x} \phi\right|_{x=0}=0$. One exploits the symmetry of (2.1) under $\phi \rightarrow-\phi$ and $x \rightarrow-x$, and simply takes a two soliton solution on $(-\infty, \infty)$ where one soliton is a mirror image of 
the other through $x=0$ [12]. If one does this with a double-kink solution then it satifies the foregoing Dirichlet condition, while the kink-anti-kink solution satisfies the Neumann condition. It is also not hard to guess how one can go beyond this solution: For $M=\infty$, the boundary condition (2.2) reduces to $\left.\phi\right|_{x=0}=\phi_{0}$. The only way that this can be obtained from a multi-soliton solution on $(-\infty, \infty)$ is to put a third, stationary soliton at the origin.

We therefore consider the three soliton solution with $k_{1}=k, k_{2}=1 / k$ and $k_{3}=1$. That is, we consider:

$$
\begin{aligned}
\tau=1 & -\epsilon v^{2} e^{-\frac{1}{k}\left(k^{2}+1\right) x-a}-\epsilon_{0}\left(\frac{k-1}{k+1}\right)^{2} e^{-\frac{1}{2 k}(k+1)^{2} x-b} F(t) \\
& +i\left\{e^{-\frac{1}{2 k}\left(k^{2}+1\right) x} F(t)+\epsilon_{0} e^{-x-b}-\epsilon \epsilon_{0} v^{2}\left(\frac{k-1}{k+1}\right)^{4} e^{-\frac{1}{k}\left(k^{2}+k+1\right) x-a-b}\right\}
\end{aligned}
$$

where we have introduced the shorthand:

$$
\epsilon=\epsilon_{1} \epsilon_{2}, \quad \epsilon_{0}=\epsilon_{3}, \quad a=a_{1}+a_{2}, \quad b=a_{3} .
$$

The function $F(t)$ is defined by:

$$
F(t) \equiv \epsilon_{1} e^{-\frac{1}{2 k}\left(k^{2}-1\right) t-a_{1}}+\epsilon_{2} e^{\frac{1}{2 k}\left(k^{2}-1\right) t-a_{2}}
$$

This solution has $\phi=0$ at $x=\infty$, and for $k>1$ it describes a left-moving soliton moving from $x=\infty$ with a right-moving "image" starting at $x=-\infty$. There is a stationary soliton with center located at $x=-b$. Viewing this as scattering off a boundary at $x=0$ one can easily see that $a$ is the phase delay of the returned soliton. To make this more explicit, observe that $\tau$ has the following asymptotic behaviour:

$$
\begin{array}{ll}
\tau(x, t) \sim 1+i \epsilon e^{-\frac{1}{2 k}\left[\left(k^{2}+1\right) x+\left(k^{2}-1\right) t\right]-a_{1}} & x,-t \rightarrow \infty \text { with } \frac{x}{t}=-\frac{k^{2}-1}{k^{2}+1} \\
\tau(x, t) \sim 1+i \epsilon e^{-\frac{1}{2 k}\left[\left(k^{2}+1\right) x-\left(k^{2}-1\right) t\right]-a_{2}} & x, t \rightarrow \infty, \quad \text { with } \frac{x}{t}=+\frac{k^{2}-1}{k^{2}+1}
\end{array}
$$

The problem now is to first show that the $\tau$-function given by (2.6) provides a solution to the boundary value problem on $[0, \infty)$ defined by $(2.1)$ and $(2.2)$. Our second purpose is to relate the parameters $a$ and $b$ of $(2.6)$ to the parameters $M$ and $\phi_{0}$ of (2.2), thereby obtaining the classical phase delay, $a$, in terms of $M$ and $\phi_{0}$. 


\subsection{The classical phase-delay}

To summarize the computation briefly, one substitutes (2.6) into (2.2), and obtains the constraint:

$$
\begin{aligned}
{\left[\mathcal{R} e(\tau) \partial_{x} \mathcal{I} m(\tau)\right.} & \left.-\mathcal{R} e(\tau) \partial_{x} \mathcal{I} m(\tau)\right]\left.\right|_{x=0} \\
& =\left.M\left[2 \cos \left(\frac{1}{2} \phi_{0}\right) \mathcal{R} e(\tau) \mathcal{I} m(\tau)-\sin \left(\frac{1}{2} \phi_{0}\right)\left(\mathcal{R} e(\tau)^{2}-\mathcal{I} m(\tau)^{2}\right)\right]\right|_{x=0}
\end{aligned}
$$

One can solve this by brute force substitution for the real and imaginary parts of $\tau$, but it is somewhat simpler to find constants $\alpha, \beta, \gamma$ and $\delta$ such that:

$$
\begin{aligned}
& \left.\partial_{x} \mathcal{R} e(\tau)\right|_{x=0}=\left.[\alpha \mathcal{R} e(\tau)+\beta \mathcal{I} m(\tau)]\right|_{x=0} \\
& \left.\partial_{x} \mathcal{I} m(\tau)\right|_{x=0}=\left.[\gamma \mathcal{R} e(\tau)+\delta \mathcal{I} m(\tau)]\right|_{x=0} .
\end{aligned}
$$

One then finds that (2.10) can be satisfied if and only if one has $\alpha=\delta$, which indeed turns out to be true. Using this one arrives at:

$$
\begin{aligned}
& M \cos \left(\frac{1}{2} \phi_{0}\right)=-\frac{\left(k^{2}+1\right)}{k} \frac{1}{\Delta}\left\{\left(1+\epsilon v^{2} e^{-a}\right)-e^{-2 b}\left(\frac{k-1}{k+1}\right)^{2}\left[1+\epsilon v^{2}\left(\frac{k-1}{k+1}\right)^{4} e^{-a}\right]\right\} \\
& M \sin \left(\frac{1}{2} \phi_{0}\right)=-2 \epsilon_{0} \frac{(k-1)^{2}}{k} e^{-b} \frac{1}{\Delta}\left\{1+\epsilon v^{2}\left(\frac{k-1}{k+1}\right)^{2} e^{-a}\right\}
\end{aligned}
$$

where

$$
\Delta \equiv\left(1-\epsilon v^{2} e^{-a}\right)+e^{-2 b}\left(\frac{k-1}{k+1}\right)^{2}\left[1-\epsilon v^{2}\left(\frac{k-1}{k+1}\right)^{4} e^{-a}\right]
$$

It is algebraically very tedious to invert this relationship. One proceeds by eliminating $e^{-b}$, and then solving for $a$. It is very convenient to introduce a new parametrization of $M$ and $\phi_{0}$ :

$$
\begin{aligned}
& \mu \equiv M \cos \left(\frac{1}{2} \phi_{0}\right) \equiv 2 \cosh (\zeta) \cos (\eta) \\
& \nu \equiv M \sin \left(\frac{1}{2} \phi_{0}\right) \equiv 2 \sinh (\zeta) \sin (\eta)
\end{aligned}
$$

where $0 \leq \zeta<\infty$ and $-\pi<\eta \leq \pi$. (In the $(\mu, \nu)$-plane the curves of constant $\zeta$ are ellipses, while the curves of constant $\eta$ are hyperbolae whose asymptotes make an angle of $\frac{1}{2} \phi_{0}$ with the $\mu$-axis.) We then find that the phase delay is given by:

$$
a=\log \left\{-\epsilon\left(\tanh \left(\frac{\theta}{2}\right)\right)^{2}(\tanh (\theta))^{2}\left[\frac{\tanh \frac{1}{2}(\theta+i \eta) \tanh \frac{1}{2}(\theta-i \eta)}{\tanh \frac{1}{2}(\theta+\zeta) \tanh \frac{1}{2}(\theta-\zeta)}\right]^{ \pm 1}\right\}
$$

There are several things to note about this formula.

(i) The ambiguity of the \pm 1 power comes from solving a quadratic equation, and is a direct reflection of the fact that (2.2) is not invariant under $\phi \rightarrow \phi+2 \pi$ (whereas (2.1) 
is invariant under this shift). Equivalently, one can flip between the + and - powers by sending $\phi_{0} \rightarrow \phi_{0}+2 \pi$.

(ii) The argument of the log is always real and positive. The discrete parameter, $\epsilon=\epsilon_{1} \epsilon_{2}$, must be chosen to arrange this. Hence:

$$
\epsilon=+1 \text { for }-\zeta<\theta<\zeta \quad \epsilon=-1 \text { for }|\theta|>\zeta
$$

This means that a kink reflects into kink for $-\zeta<\theta<\zeta$, and reflects into an antikink for $|\theta|>\zeta$. This is consistent with the fact that Dirichlet boundary conditions $(M=\infty)$ cause a kink to reflect as a kink, whereas Neumann boundary conditions $(M=0)$ cause a kink to reflect as an anti-kink. Note that these two domains of parameter space (in which a kink reflects differently) are separated from one another by a logarithmic singularity in the classical phase delay.

(iii) The choice of the power \pm 1 in (2.15) is correlated with the parameter $\eta$ and whether there is a kink, or anti-kink at the origin. Specifically, we have:

$$
\epsilon_{0}= \pm \operatorname{sign}\left(\tan \left(\frac{\eta}{2}\right)\right)
$$

where the \pm is the same as that of (2.15).

(iv) In the $M \rightarrow \infty$, or Dirichlet, limit we see that:

$$
\zeta \sim \log (M), \quad \eta \sim \frac{1}{2} \phi_{0} \quad \text { and } \quad \epsilon=-1
$$

and the phase delay collapses to:

$$
a=\log \left\{\left(\tanh \left(\frac{\theta}{2}\right)\right)^{2}(\tanh (\theta))^{2}\left[\tanh \frac{1}{2}\left(\theta+i \frac{\phi_{0}}{2}\right) \tanh \frac{1}{2}\left(\theta-i \frac{\phi_{0}}{2}\right)\right]^{ \pm 1}\right\} .
$$

In this limit 2.2 enforces Dirichlet boundary conditions. It is, however, important to note that there are two possible distinct boundary values: $\left.\phi\right|_{x=0}=\phi_{0}$ and $\left.\phi\right|_{x=0}=$ $\phi_{0}+2 \pi$. Since the boundary potential is $-M \cos \left(\frac{1}{2}\left(\phi-\phi_{0}\right)\right)$, one sees that $\left.\phi\right|_{x=0}=\phi_{0}$ is stable, while $\left.\phi\right|_{x=0}=\phi_{0}+2 \pi$ is unstable.

From now on we consider only the stable boundary value that corresponds to the positive sign in (2.19). Then one has from (2.17)

$$
\epsilon_{0}=-\operatorname{sign}\left(\tan \left(\left.\frac{1}{4} \phi\right|_{x=0}\right)\right) \text {. }
$$

It is essential to observe that we have taken $\phi_{x=\infty}=0, a b$ initio. For different boundary conditions at $x=\infty$, one should replace $\phi_{0}$ by $\left.\phi\right|_{x=0}-\left.\phi\right|_{x=\infty}$. This physical parameter is defined $\bmod 4 \pi$. An independent derivation of time delay for the Dirichlet boundary conditions, along with further details, are provided in the Appendix. 


\subsection{Boundary breather solutions}

To fully understand the semi-classical scattering computation one also needs another class of classical solutions, which we call here "boundary breathers". It is well-known that "breathers" represent bound states in the soliton-anti-soliton channel in the bulk sineGordon theory. In the same way that the classical bulk breathers can be obtained from the appropriate solution by analytic continuation of $\theta$ to imaginary axis, one might expect that the same procedure would give boundary breathers on the half-line. To see this, we set $\theta=i \vartheta\left(0<\vartheta<\frac{\pi}{2}\right)$ in the formula (A1) (see appendix). Next, we impose the following conditions for a solution to be boundary breather:

a) it should be a real function,

b) it should have finite energy and

c) the asymptotic value at $x=+\infty$ must be fixed and equal to $2 \pi n$ ( $n$-integer number).

The three-soliton (resp. soliton-anti-soliton-soliton) configuration satisfies the first condition provided that $\vartheta<-\frac{\phi_{0}}{2}$ (resp. $\vartheta<\frac{\phi_{0}}{2}$ ). However, the other conditions are satisfied by the soliton-anti-soliton-soliton configuration only, which one could have foreseen from the analogy with the bulk theory. The boundary breather solution has the form (see figure 2):

$$
\begin{aligned}
& \phi_{b}=2 \pi- \\
& -4 \arctan \frac{2 \cot \vartheta \cot \frac{\vartheta}{2} \sqrt{K} \cot \frac{\phi_{0}}{4} e^{x+x \cos \vartheta} \cos (t \sin \vartheta)+e^{2 x \cos \vartheta} K \cot ^{2} \frac{\vartheta}{2}+1}{2 \cot \vartheta \cot \frac{\vartheta}{2} \sqrt{K} e^{x \cos \vartheta} \cos (t \sin \vartheta)+e^{x} \cot \frac{\phi_{0}}{4}\left(e^{2 x \cos \vartheta} K+\cot ^{2} \frac{\vartheta}{2}\right)},
\end{aligned}
$$

where

$$
K=\cot \left(\frac{\phi_{0}}{4}-\frac{\vartheta}{2}\right) \cot \left(\frac{\phi_{0}}{4}+\frac{\vartheta}{2}\right)
$$

So, we have a continuum of classical boundary bound states when $0<\vartheta<\frac{\phi_{0}}{2}<\frac{\pi}{2}$ and for other $\phi_{0}$ according to the $2 \pi$-periodicity. In the quantum theory this continuum shrinks into a discrete set of bound states (see next section). Note that in the limit $\vartheta \rightarrow \frac{\phi_{0}}{2}$ the boundary breather (2.21) reduces to the ground state, figure 3 , and the phase delay has singularities at $\theta= \pm \frac{i}{2} \phi_{0}$. An analogous picture of bound states occurs for the anti-soliton scattering with fixed boundary conditions. 


\subsection{General solutions, integrability and Bäcklund transformations}

Thus far we have only applied the method of images to obtain certain special classical solutions of the boundary sine-Gordon problem (1.1). It is natural to suggest that general solutions of (1.1) can be obtained by similar methods. This in turn would establish the classical integrability the boundary problem (1.1). It is, in fact, rather straightforward to show that both of these conclusions are true, at least for the problem (1.1) with $\phi_{0}=0$. The method we will employ can almost certainly be generalized to problems with $\phi_{0} \neq 0$, and also has the virtue that it can be used to construct the integrable boundary potentials for the more general Toda models. A related approach has been followed in [13, 14. The basic idea is to use the fact that any integrable hierarchy has Bäcklund transformations: that is, solutions can be mapped into one another by non-trivial gauge transformations constructed from the affine Lie algebra action on the corresponding LAX system. For the sine-Gordon equation, the requisite Bäcklund transformation can be cast in the following form:

$$
\partial_{u}(\phi+\psi)=e^{\zeta} \sin \left(\frac{\phi-\psi}{2}\right) ; \quad \partial_{v}(\phi-\psi)=e^{-\zeta} \sin \left(\frac{\phi+\psi}{2}\right)
$$

where $u=x-t, v=x+t$, and $\zeta$ is an arbitrary constant parameter. The point is that $\phi$ satisfies the sine-Gordon equation (2.1) if and only if $\psi$ does so as well. Suppose that $\psi$ is a solution to sine-Gordon on $[0, \infty)$ satisfying a Dirichlet boundary condition: $\left.\psi\right|_{x=0}=2 \eta$, where $\eta$ is a constant. It follows immediately from (2.22) that $\phi$ is a solution to sine-Gordon satisfying (2.2), where $M$ and $\phi_{0}$ are given in terms of $\zeta$ and $\eta$ by (2.14). Thus, if one can solve the Dirichlet problem, one can solve the more general problem by a Bäcklund transformation.

Observe that if $\eta=0$, or equivalently $\phi_{0}=0$, then the Dirichlet problem can be solved trivially by method of images: one gets the solution on the half-line by extending it as an odd function on the full line. Thus, the Bäcklund transformation essentially

defines the generalized method of images. It is also by no means an accident that the parameters entering into the Bäcklund transformation $(\eta$ and $\zeta$ ) are precisely the rapidity parameters that turn up in the phase delay (2.15). One should also note that the form of the integrable boundary potential is given directly by the Bäcklund transformation. This fact should easily generalize to Toda systems [15].

Bäcklund transformations, in general, are invertible transformations on the solution space of an integrable hierarchy. The simplest forms of them modify the constants of 
the motion of solution, and possibily add or subtract a soliton. One can certainly find a Bäcklund transformation that will introduce a stationary soliton into the soliton-soliton solution of sine-Gordon. As a result, the general three soliton solution employed above can be obtained from the two soliton solution that is appropriate for the "trivial" Dirichlet problem with $\phi_{0}=0$. We therefore expect that any solution of the trivial Dirichlet problem can be mapped onto the generic problem (1.1), thus establishing the classical integrability. Here we shall content ourselves with having explicitly established these results for $\phi_{0}=0$, and having shown that there is a direct link between the form of the potential and the structure of the underlying integrable hierarchy.

\section{The semi-classical analysis}

We start the discussion by considering the $\beta^{2} \rightarrow 0$ limit of the results in [7]. The (quantum) boundary S matrix elements are [7]

$$
\begin{aligned}
& S_{+}^{+}(\theta) \equiv P_{+}(\theta)=\cos [\xi-(t-1) i \theta] R(\theta) \\
& S_{-}^{-}(\theta) \equiv P_{-}(\theta)=\cos [\xi+(t-1) i \theta] R(\theta),
\end{aligned}
$$

and

$$
S_{+}^{-} \equiv Q_{+}(\theta)=S_{-}^{+}=Q_{-}(\theta)=\frac{k}{2} \sin [2(t-1) i \theta] R(\theta)
$$

where $\theta$ is the rapidity of an incoming particle. The parameter $t$ is defined by:

$$
t=\frac{8 \pi}{\beta^{2}}
$$

The function $R(\theta)$ decomposes as

$$
R(\theta)=R_{0}(\theta) R_{1}(\theta)
$$

where $R_{0}$ is a normalization factor ensuring unitarity and crossing symmetry that does not depend on the boundary conditions. The dependence upon boundary conditions appears in $R_{1}$, which reads

$$
R_{1}(\theta)=\frac{1}{\cos \xi} \sigma(\eta, \theta) \sigma(i \Theta, \theta) .
$$

Two of the four parameters $k, \xi, \eta, \Theta$ are independent, and we have the relations

$$
\cos \eta \cosh \Theta=\frac{1}{k} \cos \xi, \quad \cos ^{2} \eta+\cosh ^{2} \Theta=1+\frac{1}{k^{2}}
$$


These parameters are related with $M$ and $\varphi_{0}$ in an unknown way. An expression for the functions $R_{0}$ and $\sigma$ involving infinite products of $\Gamma$-functions is given in [7]. There are also simple integral representations:

$$
\sigma(\xi, \theta)=\frac{\cos \xi}{\cos [\xi-i(t-1) \theta]} \exp \left\{\int_{-\infty}^{\infty} \frac{d x}{x} \frac{\sinh \left(t-1+\frac{2 \xi}{\pi}\right) x}{2 \cosh (t-1) x \sinh x} e^{i \frac{2}{\pi}(t-1) \theta x}\right\},
$$

and

$$
R_{0}(\theta)=\exp \left\{-\int_{-\infty}^{\infty} \frac{d x}{x} \quad \frac{\sinh \frac{3}{2}(t-1) x \sinh \left(\frac{t}{2}-1\right) x}{\sinh \frac{x}{2} \sinh 2(t-1) x} e^{i \frac{2}{\pi}(t-1) \theta x}\right\} .
$$

One has $\sigma(\xi, \theta)=\sigma(-\xi, \theta)$. The only difference between the scattering of solitons and anti-solitons arises therefore from the pre-factor $\cos [\xi \mp i(t-1) \theta]$ in (3.1).

For simplicity we consider only the limit in which $M \rightarrow \infty$. One can identify the corresponding values $k=0$ and $\Theta=\infty$ easily since, at these values, the topological charge $Q=\frac{\beta}{2 \pi} \int_{0}^{\infty} \partial_{x} \varphi d x$ is conserved and therefore the amplitudes $Q_{ \pm}$must vanish. One has also $\eta=\xi$ so

$$
R_{1}(\theta)=\frac{1}{\cos \xi} \sigma(\xi, \theta)
$$

Consider now the quantum boundary $\mathrm{S}$ matrix at the leading order in $\frac{1}{\beta^{2}}$ as $\beta \rightarrow 0$. The computation is most easily done by using the integral representation given above, and evaluating the integrals explicitly by the residue theorem. This provides convergent expressions where the $\beta \rightarrow 0$ limit can be investigated term by term. To get non-trivial results one must scale $\xi$ as

$$
\frac{\beta^{2}}{8 \pi} \xi \rightarrow \hat{\xi}
$$

We find then

$$
P_{ \pm}(\theta)=\exp \left( \pm \frac{8 i \pi \hat{\xi} \kappa}{\beta^{2}}+\frac{8 i \pi|\hat{\xi}| \kappa}{\beta^{2}}\right) \frac{\mathcal{S}(\theta ; 0)[\mathcal{S}(2 \theta ; 0)]^{1 / 2}}{[\mathcal{S}(\theta ; \hat{\xi}) \mathcal{S}(\theta ;-\hat{\xi})]^{1 / 2}}
$$

where

$$
\mathcal{S}(\theta ; y)=\exp \left(\frac{8 i}{\beta^{2}} \int_{0}^{\theta} d v \ln \tanh ^{2} \frac{v+i y}{2}\right)
$$

$\mathcal{S}(\theta ; 0)$ being the semi-classical approximation to the bulk soliton-soliton S-matrix [8] (in the following we denote it also as $\mathcal{S}(\theta)$ ), and $\kappa=\operatorname{sign} \theta$ (in the following we assume that $\theta>0)$.

Before discussing the relation between formula (3.9) and the classical computations of the preceding section, it is useful to comment on the bound states of the quantum theory. 
Poles of the $R_{0}$ term are associated with breathers and do not correspond to the boundary (new) bound states. The latter correspond rather to poles of the $\sigma(\xi, \theta)$ term which are located in the physical strip $\operatorname{Im} \theta \in[0, \pi / 2]$. By inspection of the $\Gamma$-product expression [7] one finds two families of poles of $\sigma$ :

$$
\begin{aligned}
& \theta_{n, l}^{(1)}=-i \frac{\pi}{t-1}\left(n+\frac{1}{2}\right) \pm i \frac{\xi}{t-1}-2 i l \pi \\
& \theta_{n, l}^{(2)}=i \frac{\pi}{t-1}\left(n+\frac{1}{2}\right) \pm i \frac{\xi}{t-1}+(2 l+1) i \pi
\end{aligned}
$$

where $n, l$ are integers. Let us restrict to $\xi>0$. Then the only physical poles are those that correspond to $+\operatorname{sign}$ in $\theta^{(1)}$ and $-\operatorname{sign}$ in $\theta^{(2)}$. The first pole that enters the physical strip (from the bottom) is $\theta_{0,0}^{(1)}$ for $\xi \geq \frac{\pi}{2}$. The number of poles of $\sigma$ in the physical strip increases monotonically with $\xi$ for $\xi$ small enough, and as $t$ gets large it becomes simply of the form $\xi / \pi$. These poles are cancelled by the cosine term in $P_{-}$and therefore appear only in the $P_{+}$amplitude. As $\xi$ reaches the value $\xi=\frac{4 \pi^{2}}{\beta^{2}}$ the poles densely fill the interval $0<\operatorname{Im} \theta<\frac{\pi}{2}$ and a pole at $\theta=i \pi / 2$ appears corresponding to the emission of a zero momentum soliton. As argued in [7] this corresponds to a change in the ground state of the system. For $0<\varphi_{0}<\frac{\pi}{\beta}$ the ground state is $\varphi \rightarrow 0$ at infinity but for $\frac{\pi}{\beta}<\varphi_{0}<3 \frac{\pi}{\beta}$ it is $\varphi \rightarrow \frac{2 \pi}{\beta}$ at infinity. Therefore the value $\xi=\frac{4 \pi^{2}}{\beta^{2}}$ corresponds to $\varphi_{0}=\frac{\pi}{\beta}$ and it is the upper physical value for $\xi$ : as $\varphi_{0}$ varies arbitrarily, $\xi$ never gets larger than $\frac{4 \pi^{2}}{\beta^{2}}$ and the set of poles $\theta_{n, l}^{(2)}$ never enters the physical strip. Observe that there are bound states in the quantum theory when in the classical case the kink sitting in the middle and the incoming one are of opposite topological charges in complete agreement with the discussion of boundary breathers in section 2 .

To establish the relation between (3.9) and (2.19), first recall [8] the general relation between the quasi-classical scattering phase shift, $\delta(\theta)$, and the classical phase shift, $a(\theta)$ :

$$
\delta(\theta)=\text { constant }+\frac{m}{2} \int_{0}^{\theta} a(\eta) d \eta
$$

Here, $m$ is the classical mass of the particles involved in the scattering. Using the "semiclassical Levinson theorem" to determine the constant in this formula we deduce, using (2.19), the relation

$$
P_{+}(\theta) \equiv e^{2 i \delta(\theta)}
$$

where

$$
2 \delta(\theta)=2 n_{B} \pi+\frac{8}{\beta^{2}} \int_{0}^{\theta} d \eta \log \frac{\tanh ^{2} \eta \tanh ^{2} \frac{\eta}{2}}{\tanh \frac{1}{2}\left(\eta+i \frac{\beta \varphi_{0}}{2}\right) \tanh \frac{1}{2}\left(\eta-i \frac{\beta \varphi_{0}}{2}\right)}
$$


and $n_{B}$ is the number of bound states. Since according to the preceeding discussion $n_{B}=\frac{\xi}{\pi}=\frac{8 \hat{\xi}}{\beta^{2}}$ for $0<\xi<\frac{4 \pi^{2}}{\beta^{2}}$ as $\beta \rightarrow 0$ we see that formula (3.9) is in complete agreement with the classical phase shift (2.19) and that $\xi$ is proportional to $\phi_{0}$. In the region $-\frac{4 \pi^{2}}{\beta^{2}}<\xi<0$ there are no physical poles for $P_{+}$and $n_{B}=0$. A similar discussion can be carried out for $\xi<0$ and $P_{-}$. As $\beta \rightarrow 0$ the number of physical poles of $P_{-}$varies as $n_{B}=-\frac{\xi}{\pi}=-\frac{8 \hat{\xi}}{\beta^{2}}$ and again we have agreement with the semi-classical Levinson theorem.

From comparison of (3.9) and the classical phase shift we see that $\xi$ and $\varphi_{0}$ are related linearly as $\xi=\frac{4 \pi}{\beta} \varphi_{0}$. This leads correctly to the emission of a zero momentum soliton with the ground state degeneracy as discussed in [7]. This linear relation can hold only for $|\xi|<\frac{4 \pi^{2}}{\beta^{2}}$. Beyond that value the ground state changes. To compare the quantum result with the classical phase shift we must then correlate appropriately the value of $\phi$ at infinity in the latter. The net effect is to replace $\phi_{0}$ by $\phi_{0}-2 \pi$. Eventually, the variation of $\xi$ with $\varphi_{0}$ is therefore

$$
\xi=\frac{4 \pi}{\beta}\left(\varphi_{0}-\frac{2 \pi}{\beta} \operatorname{Int}\left[\frac{\beta \varphi_{0}}{2 \pi}+\frac{1}{2}\right]\right),
$$

as illustrated in figure 4 . It is very likely that (3.15) is exactly true for finite $\beta$ as well.

We can finally recover (3.9) without appealing to our knowledge of quantum boundary bound states. For this one has to evaluate the action for the classical configuration. Following the discussion in [8] we have,

$$
2 \delta(\theta)=C\left(\varphi_{0}\right)+\frac{8}{\beta^{2}} \int_{0}^{\theta} d \eta \log \frac{\tanh ^{2} \eta \tanh ^{2} \frac{\eta}{2}}{\tanh \frac{1}{2}\left(\eta+i \frac{\beta \varphi_{0}}{2}\right) \tanh \frac{1}{2}\left(\eta-i \frac{\beta \varphi_{0}}{2}\right)}, \quad \text { if } \quad\left|\beta \varphi_{0}\right|<\pi,
$$

where we used the soliton mass $m=\frac{8}{\beta^{2}}$, and $\delta$ satisfies the differential equation

$$
\delta(\theta)-\tanh \theta \delta^{\prime}(\theta)=\int_{0}^{+\infty} d t\left(\int_{0}^{+\infty} d x \dot{\varphi}^{2}-8 \sinh \theta \tanh \theta\right) .
$$

We restrict to the situation where we send in a kink, and $\varphi_{0}$ is positive and so there is an anti-kink at the origin (see figure 1). In this case there are quantum boundary bound states. It is difficult to perform the double integral for the three-soliton solution explicitly because of the cumbersome expression for the integrand (A1). One might hope that in order to find the $\theta$-independent piece of the phase shift it is sufficient to evaluate both sides of (3.17) in the limit as $\theta \rightarrow+\infty$ or $\theta \rightarrow 0$. However both of these limits do not seem to be helpful, because due to the non-uniform convergence of the right hand side 
of (3.17) it is not allowed to interchange such a limit with the integration. We evaluated the right hand side of (3.17) (with fixed $\theta$ ) numerically for several different values of $\phi_{0}$ using Mathematica. Combining (3.16) and (3.17), we obtain an estimate for $C\left(\varphi_{0}\right)$, in agreement with $C\left(\varphi_{0}\right)=\frac{8 \pi}{\beta} \varphi_{0}$ with accuracy $0.1 \%$. This result was checked for different values of $\theta$. We therefore obtain agreement with the semi-classical Levinson theorem.

It is also interesting to investigate the cross-unitarity relation of [7] in this semiclassical limit. In general we have

$$
P_{+}\left(\frac{i \pi}{2}-\theta\right)=S_{T}(2 \theta) P_{-}\left(\frac{i \pi}{2}+\theta\right)+S_{R}(2 \theta) P_{+}\left(\frac{i \pi}{2}+\theta\right)
$$

where $S$ are the bulk $\mathrm{S}$ matrix elements. In the semi-classical limit we are considering, the reflection amplitude $S_{R}$ vanishes. In this same limit we use (3.9) to find

$$
\begin{aligned}
\frac{P_{+}\left(\frac{i \pi}{2}-\theta\right)}{P_{-}\left(\frac{i \pi}{2}+\theta\right)}= & \frac{\mathcal{S}\left(\frac{i \pi}{2}-\theta\right)}{\mathcal{S}\left(\frac{i \pi}{2}+\theta\right)}\left[\frac{\mathcal{S}\left(\frac{i \pi}{2}-\theta+i \hat{\xi}\right) \mathcal{S}\left(\frac{i \pi}{2}-\theta-i \hat{\xi}\right)}{\mathcal{S}\left(\frac{i \pi}{2}+\theta+i \hat{\xi}\right) \mathcal{S}\left(\frac{i \pi}{2}+\theta-i \hat{\xi}\right)}\right]^{-1 / 2} \\
& {\left[\frac{\mathcal{S}(i \pi-2 \theta)}{\mathcal{S}(i \pi+2 \theta)}\right]^{1 / 2} }
\end{aligned}
$$

We simplify this expression by using the following relations deduced from unitarity and crossing symmetry at this order [16]

$$
\begin{aligned}
S\left(\frac{i \pi}{2}+\theta\right) & =S_{T}\left(\frac{i \pi}{2}-\theta\right) \\
S(\theta) & =\frac{1}{S(-\theta)} \\
\mathcal{S}(\theta) & =e^{-i \kappa \frac{8 \pi^{2}}{\beta^{2}}} \mathcal{S}_{T}(\theta),
\end{aligned}
$$

to obtain (3.18) indeed.

\section{Conclusions}

Although the method of images works nicely in the classical theory, it does not seem to extend to the quantum case: we have not been able to recast the boundary $\mathrm{S}$ matrix of [7] as a product of bulk S-matrix elements. A related phenomenon is the non-trivial structure of the boundary S-matrix with one-loop corrections (the semi-classical expressions being the tree approximation). Recall that in the bulk one has

$$
\mathcal{S}_{1}(\theta ; y)=\exp \left(\frac{8 i}{\beta^{\prime 2}} \int_{0}^{\theta} d v \ln \tanh ^{2} \frac{v+i y}{2}\right)
$$


where

$$
\beta^{2} \equiv \beta^{2} \frac{8 \pi}{8 \pi-\beta^{2}}=\frac{8 \pi}{t-1}
$$

Scaling

$$
\frac{\beta^{\prime 2}}{8 \pi} \xi \rightarrow \hat{\xi}_{1}
$$

one finds the following next-to-leading correction to the boundary S-matrix in the Dirichlet problem:

$$
P_{ \pm}(\theta) \approx \exp \left( \pm \kappa \frac{8 i \pi \hat{\xi}_{1}}{\beta^{\prime 2}}+\kappa \frac{8 i \pi\left|\hat{\xi}_{1}\right|}{\beta^{\prime 2}}\right) \frac{\mathcal{S}_{1}(\theta)\left[\mathcal{S}_{1}(2 \theta)\right]^{1 / 2}}{\left[\mathcal{S}_{1}\left(\theta ;-\hat{\xi}_{1}\right) \mathcal{S}_{1}\left(\theta ; \hat{\xi}_{1}\right)\right]^{1 / 2}}\left[\tanh \left(\frac{\theta}{2}-i \frac{\pi}{4}\right)\right]^{1 / 2}
$$

In addition to the usual replacement of $\beta^{2}$ by $\beta^{\prime 2}$ we see the appearance of an entirely new factor involving the square-root of a hyperbolic tangent.

It would be interesting to investigate further the structure of boundary bound states, most particularly in the quantum theory. Also, besides the semi-classical limit, one could also take the non-relativistic limit of the S-matrix. The appropriate candidate for the resulting non-relativistic potential would be probably the exactly-solvable Pöschl-Teller model on half-line [17]:

$$
U(x)=U_{0}\left(\frac{\mu(\mu-1)}{\sinh ^{2} \alpha x}-\frac{\nu(\nu-1)}{\cosh ^{2} \alpha x}\right)
$$

where $\alpha, \mu, \nu$ and $U_{0}$ are some constants, related to the parameters $\beta$ and $\varphi_{0}$ of sineGordon model. Note that (4.4) is simply a superposition of the repulsive hard-core kinkkink potential (first term) and the kink-antikink potential (second term), which are known from the bulk theory [16]. This problem will be studied elsewhere [18].

\section{Acknowledgements:}

We would like to thank P. Fendley for interesting discussions and A.Kapustin for the help with numerical computation and many useful remarks. N.W. would like to thank the Theory Division at CERN for its hospitality while this work was completed. This work was supported by the Packard foundation, the National Young Investigator program (NSF-PHY-9357207) and the DOE under grant number DE-FG03-84ER40168.

\section{Appendix}

We give here the explicit general form of classical solutions and directly extract the time delay $(2.19)$ in the limit $M=\infty$. 
In the center of mass reference frame the solution to (2.1) reads:

$$
\phi=\mp 4 \arctan \frac{2 e^{x \operatorname{ch} \theta-a_{1}} \operatorname{ch}(t \operatorname{sh} \theta) \pm e^{x-a_{3}} \mp e^{x(1+2 \operatorname{ch} \theta)-2 a_{1}-a_{3}+2 \log \left(u^{2} v\right)}}{2 e^{x(1+\operatorname{ch} \theta)-a_{1}-a_{3}+2 \log (u)} \operatorname{ch}(t \operatorname{sh} \theta) \pm e^{2 x \operatorname{ch} \theta-2 a_{1}+2 \log (v)} \mp 1},
$$

where $u=\tanh \left(\frac{\theta}{2}\right), v=\tanh (\theta)$, and we have set $a_{1}=a_{2}$, which means that the solution is invariant under the transformation $t \rightarrow-t$. The upper (resp. lower) sign corresponds to the situation when a stationary soliton (resp. anti-soliton) is used to adjust the value of field at the boundary. Solution (A1) refers to the case when the incoming and outgoing particle is the soliton with asymptotic value $\phi=2 \pi$ at $x=+\infty$.

Let us represent (A1) in the form of rational function of variable $\operatorname{ch}(t \operatorname{sh} \theta)$ :

$$
\phi(x, t)=4 \arctan \frac{r_{2}+s_{2} \operatorname{ch}(t \operatorname{sh} \theta)}{r_{1}+s_{1} \operatorname{ch}(t \operatorname{sh} \theta)} .
$$

The condition $\phi(x=0, t)=\phi_{0}$ implies that

$$
\frac{r_{2}}{r_{1}}=\frac{s_{2}}{s_{1}}=\tan \left(\frac{\phi_{0}}{4}\right)
$$

from which follows immediately

$$
a_{3}=\log \left(\mp u^{2} \tan \frac{\phi_{0}}{4}\right)
$$

For the argument of logarithm to be positive one should take $\phi_{0}>0$ with the stationary anti-soliton and $\phi_{0}<0$ with the stationary soliton. This is illustrated in figure 1 . Further, we obtain from (A2)

$$
2 a_{1}=\log \left[u^{2} v^{2} \frac{u^{2}+\tan ^{2}\left(\frac{\phi_{0}}{4}\right)}{1+u^{2} \tan ^{2}\left(\frac{\phi_{0}}{4}\right)}\right],
$$

which agrees with (2.19) where $a=2 a_{1}$. Note that the time delay, obtained by (A3), is in fact always a time advance in both the attractive and repulsive cases. For the same value of $\phi_{0}$ the time delay for the soliton that lives on the left half-line $x<0$ is not the same as that of the right half-line soliton (except for $\phi_{0}= \pm \pi$ ). It differs by the sign of power factor in the formula (2.19). The position of the "left" soliton is not the exact mirror image of the "right" soliton for generic $\phi_{0}$. 


\section{References}

[1] A.O. Caldeira, A.J. Leggett, Physica 121A (1983) 587.

[2] I. Affleck, A. Ludwig, Nucl. Phys. B360 (1991) 641.

[3] C.Kane, M.Fisher, Phys. Rev. B46 (1992) 15233.

[4] P. Fendley, A. Ludwig, H. Saleur, "Exact Hall conductance through point contacts in the $\nu=1 / 3$ fractional quantum Hall effect", preprint USC-94-12.

[5] C. Callan, I. Klebanov, Phys. Rev. Lett. 72 (1994) 1968.

[6] P. Fendley, H. Saleur, N.P. Warner, "Exact solution of a massless scalar field with a relevant boundary interaction", preprint USC-94-10.

[7] S. Ghoshal and A.B. Zamolodchikov, "Boundary State and Boundary $S$ Matrix in Two-Dimensional Integrable Field Theory", RU-93-20, hep-th/9306002.

[8] R. Jackiw, G. Woo, Phys. Rev. D12 (1975) 1643.

[9] V. Korepin, Th. and Math. Phys. 34 (1978) 1.

[10] J. Cardy, Nucl. Phys. B240 (1984) 514.

[11] M. Ablowitz, H. Segur "Solitons and the inverse scattering transform", SIAM Studies in Appl. Math. 1981.

[12] R.M. Deleonardis, S.E. Trullinger and R.F. Wallis, J. Appl. Phys. 51 (1980) 1211-1226;

[13] E.K. Sklyanin, Funct. Anal. Appl. 21 (1987) 164.

[14] V.O. Tarasov, Inverse Problems 7 (1991) 435.

[15] E. Corrigan, P.E. Dorey, R.H. Rietdijk, R. Sasaki, "Affine Toda field theory on a half line", preprint 1994, hep-th/9404108.

[16] A.B. Zamolodchikov, Al.B. Zamolodchikov, Ann. Phys. 120 (1979), 253.

[17] G. Pöschl, E. Teller, Z. Physik 83 (1933) 143.

[18] A.Kapustin, S.Skorik, in preparation. 


\section{Figure captions}

Figure 1: a solution of the classical sine-Gordon equation with fixed boundary conditions. For $\left.\phi\right|_{x=0}=\phi_{0}=\frac{3 \pi}{8}$ the solution is constructed out of a left-moving soliton, its rightmoving image at $x<0$ and the stationary anti-soliton in the middle (upper graph). For $\left.\phi\right|_{x=0}=\phi_{0}=-\frac{3 \pi}{8}$ the solution is built out of three solitons (lower graph).

Figure 2: a boundary bound state (boundary breather) for the values $\theta=\frac{i \pi}{6}$ and $\phi_{0}=\frac{3 \pi}{4}$ at four different instants of time.

Figure 3: two possible ground state configurations for $\phi_{0}=\frac{3 \pi}{4}$. The configuration with asymptotic behaviour $\phi \rightarrow 0$ at infininty has lower energy than the other one.

Figure 4: variation of $\xi$ with $\phi_{0}$. 
This figure "fig1-1.png" is available in "png" format from: http://arXiv.org/ps/hep-th/9408004v2 


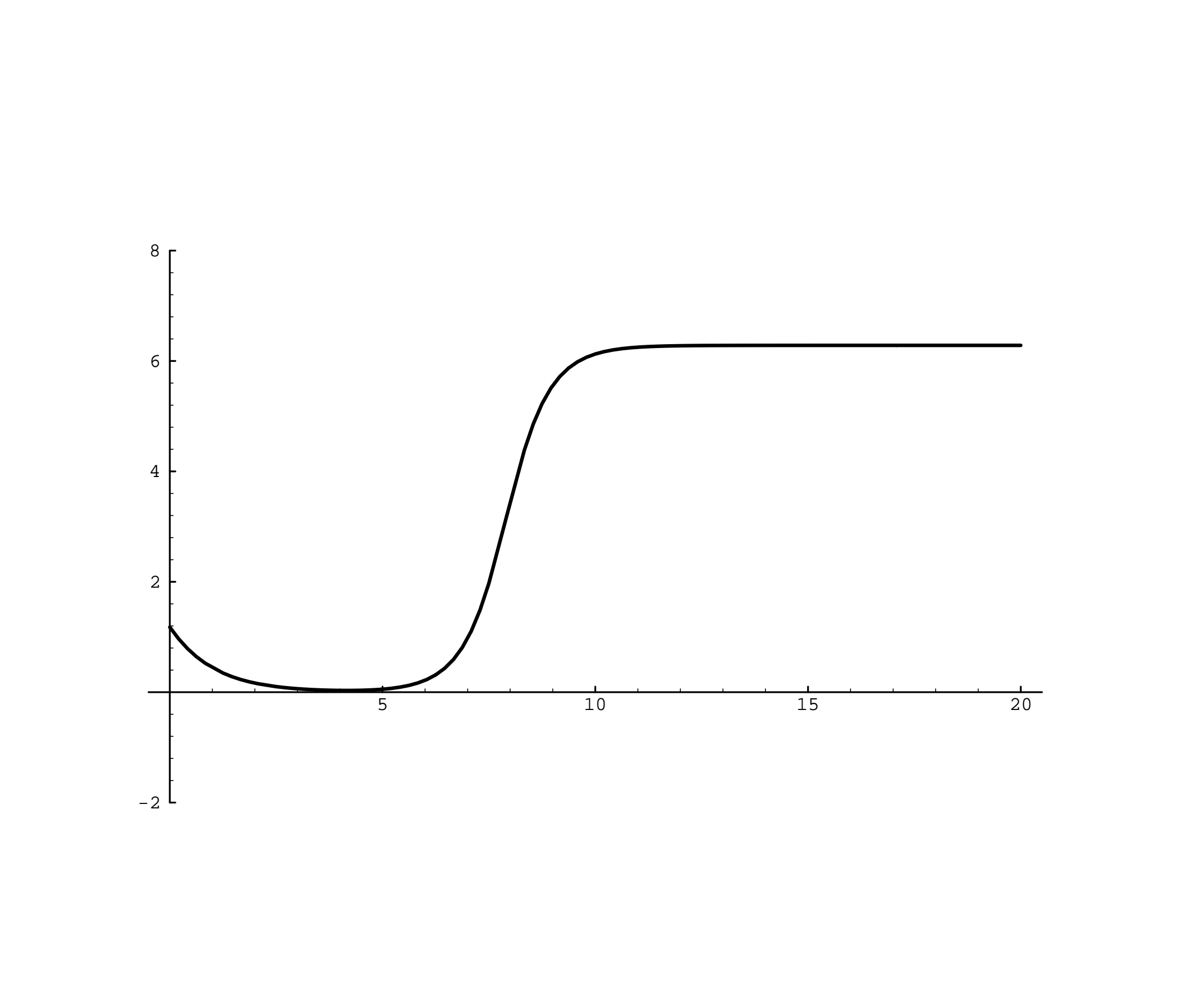


This figure "fig1-2.png" is available in "png" format from: http://arXiv.org/ps/hep-th/9408004v2 


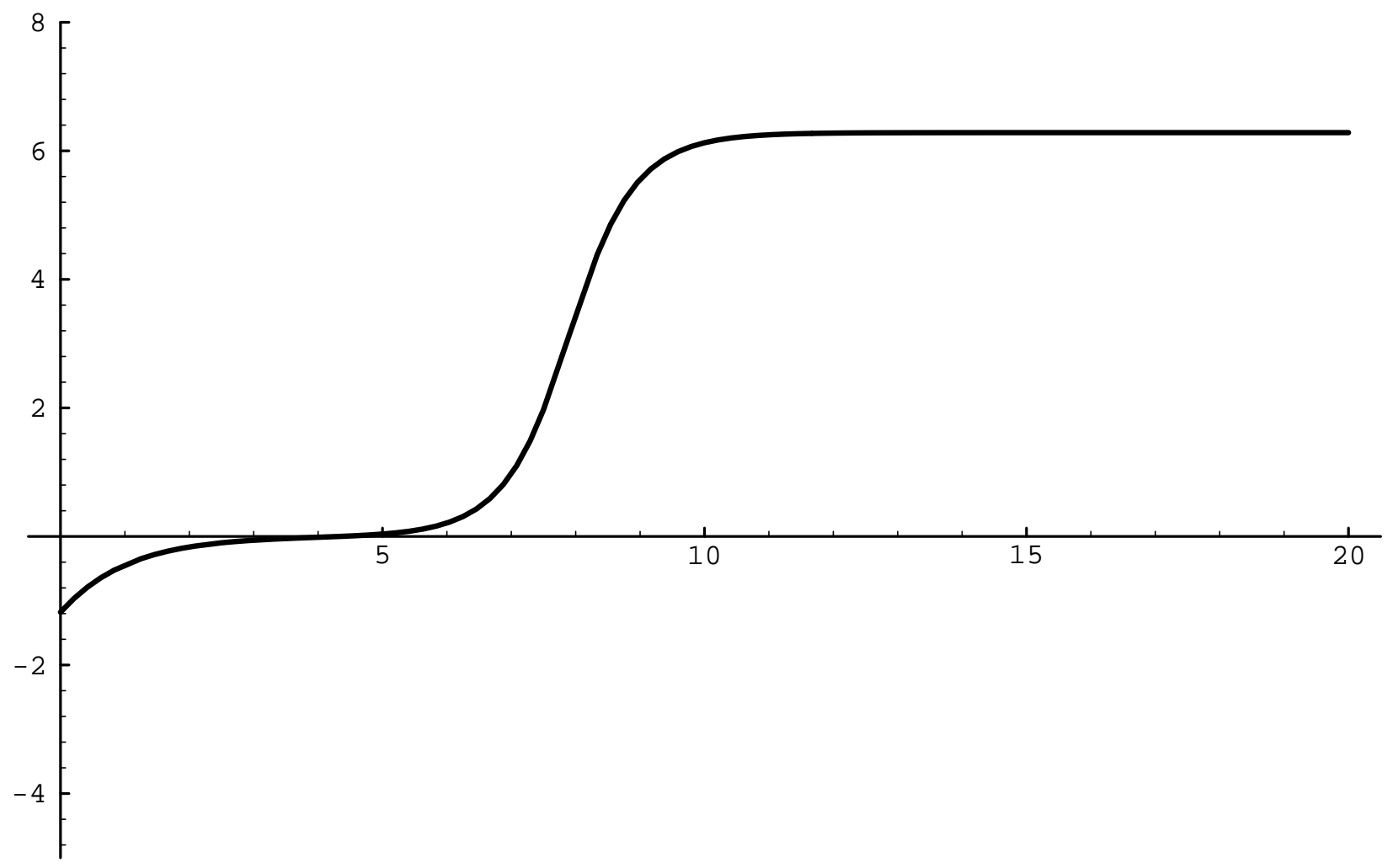


This figure "fig1-3.png" is available in "png" format from: http://arXiv.org/ps/hep-th/9408004v2 


$$
C
$$


This figure "fig1-4.png" is available in "png" format from: http://arXiv.org/ps/hep-th/9408004v2 


$$
\sqrt{6}
$$


This figure "fig1-5.png" is available in "png" format from: http://arXiv.org/ps/hep-th/9408004v2 


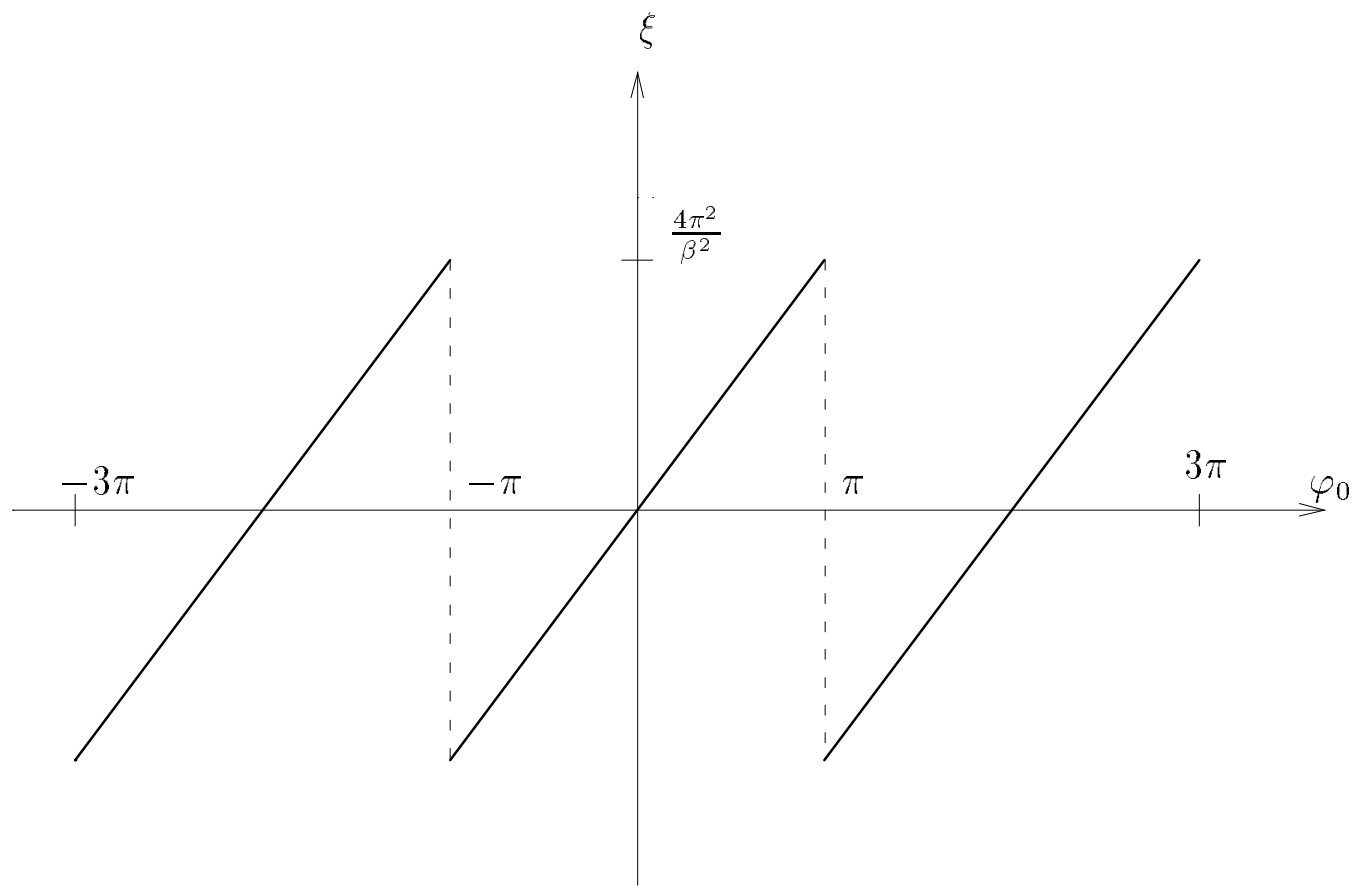

Figure 4: Variation of $\xi$ as a function of $\varphi_{0}$. 